\title{
Methodology for characterisation of glass fibre composite architecture
}

\author{
Hansen, Jens Zangenberg; Larsen, J.B.; Østergaard, R.C.; Brøndsted, Povl
}

Published in:

Plastics, Rubber \& Composites

Link to article, DOI:

10.1179/1743289811Y.0000000067

Publication date:

2012

Link back to DTU Orbit

\section{Citation (APA):}

Hansen, J. Z., Larsen, J. B., Østergaard, R. C., \& Brøndsted, P. (2012). Methodology for characterisation of glass fibre composite architecture. Plastics, Rubber \& Composites, 41(4/5), 187-193.

https://doi.org/10.1179/1743289811Y.0000000067

\section{General rights}

Copyright and moral rights for the publications made accessible in the public portal are retained by the authors and/or other copyright owners and it is a condition of accessing publications that users recognise and abide by the legal requirements associated with these rights.

- Users may download and print one copy of any publication from the public portal for the purpose of private study or research.

- You may not further distribute the material or use it for any profit-making activity or commercial gain

- You may freely distribute the URL identifying the publication in the public portal 


\title{
Methodology for Characterisation of Glass Fibre Composite Architecture*
}

\author{
J. Zangenberg ${ }^{\dagger 1,2}$, J. B. Larsen ${ }^{2}$, R. C. Østergaard ${ }^{2}$, and \\ P. Brøndsted ${ }^{1}$ \\ ${ }^{1}$ Materials Research Division, Ris $\varnothing$ DTU, Frederiksborgvej 399, 4000 \\ Roskilde, Denmark \\ ${ }^{2}$ LM Wind Power, Jupitervej 6, 6000 Kolding, Denmark
}

September 4, 2012

\begin{abstract}
The present study outlines a methodology for micro-structural characterisation of fibre reinforced composites containing circular fibres. Digital micrographs of polished cross-sections are used as input to a numerical image processing tool that determines a spatial mapping and radii detection of the fibres. The information is used for different analyses to investigate and characterise the fibre architecture. As an example, the methodology is applied to glass fibre reinforced composites with varying fibre content. The different fibre volume fractions affects the number of contact points per fibre, the communal fibre distance, and the local fibre volume fraction. The fibre diameter distribution and packing pattern remain somewhat similar for the considered materials.

The methodology is a step towards a better understanding of the composite micro-structure, and can be used to evaluate the interconnection between the fibre architecture and composite properties.
\end{abstract}

Keywords: Glass fibres; Scanning electron microscopy (SEM); Composite micro-structure; Microstructural characterisation.

\section{Introduction}

The fibre volume fraction (FVF) is considered being the most crucial microstructural parameter for describing a fibre reinforced composites. The pa-

\footnotetext{
*This paper is part of a special issue on Deformation and fracture of polymers and their composite

${ }^{\dagger}$ Corresponding author. Tel.: +45 5138 8407. Fax: +45 4677 5758. Email: jzan@risoe.dtu.dk and jezh@lmwindpower.com
} 
rameter forms the basis for the well-known rule of mixtures for determining the in-plane stiffness of a composite material, see e.g. Jones [1]. Commonly, the FVF is determined on basis of the weight fractions and the densities of the individual constituents, and yields an average laminate parameter for the entire composite. However, on a local scale the FVF attains larger values e.g. inside individual bundles where the fibre concentration is enlarged. It is evident that there exists other micro-structural parameters that characterise a fibre reinforced composite e.g. packing pattern, neighbouring distance, clustering, etc. Information about these parameters can be extracted from measurements on cross-sections of the given composite. Digital image-analysis and -processing are tools to be used to identify the material micro-structure. Attempts have been made in order to characterise the full 3 dimensional structure of (glass) fibre composites (FVF, fibre misalignment, waviness, fibre curvature, etc.) using a sectioning approach based on microscope images. This analysis requires great accuracy in the sectioning process and in indetifing the individual fibres. The method is time consuming, but serves well in order to describe the local appearance of the micro-structure. This 3D characterisation has been carried out by several researchers, see for instance Paluch [2] or Clarke et al. 3]. These studies also include determination of the fibre misalignment angle inspired by the ideas of Yurgartis [4] where the misalignment angle is determined by measurements of the elliptical fibre-shape that appears on an inclined cross section compared to the principal fibre direction. Using numerical routines, Creighton et al. [5] and Kratmann et al. 6] determined the misalignment angle for much lower resolution images, and thus saving the processing time. The difference between $2 \mathrm{D}$ and $3 \mathrm{D}$ characterisation is outlined in the, to some extend, personal account of Exner [7].

Based on digital micrographs of a planar and polished cross-section of a uni-directional fibre composite, this study presents a methodology which detects the spatial distribution and radii of the fibres. This mapping of the fibres is used to define a number of parameters that are considered relevant for a micro-structural characterisation of fibre reinforced composites. The method is capable of analysing local regions as well as entire bundles or cross-sections. For illustrating the applicability, the methodology is applied to different glass fibre reinforced composites with a varying fibre content. The ideas presented are inspired by the review work of Guild \& Summerscales [8] who discuss a number of different approaches for image analysis of fibre composites, Pyrz [9] who quantitatively investigated the composite micro-structure by statistical tools, and Paluch [2] who made a 3D characterisation based on 2D image techniques. This stereological approach for investigating the 3D micro-structure based on 2D images has been used extensively, but it seems that the methods and ideas have not been brought to any practical experiences. For instance when the composite micro-structure is investigated numerically using representative volume elements (RVE's), 
general assumptions are made on the fibre distribution and packing pattern, communal fibre distance, boundary conditions, etc. Wongsto \& Li [10] investigated the effect of boundary condition on the RVE and made numerical simulation of a random distributed fibre arrangement. The fibre randomness was further investigated by Melro et. al [11] who developed a statistically characterised algorithm to generate random composite cross-sections. The predictions from the algorithm in terms of effective composite properties agreed well with experimental data. The idea of random fibre distribution was also used by Mishnaevsky \& Brøndsted [12] who, in a numerical study, considered fatigue damage of unidirectional fibre reinforced composites. Ongoing work will extend the knowledge of the interplay between fatigue damage and fibre architecture since details in the fibre architecture has proven to be detrimental for the fatigue performance.

\section{Method}

Shape detection algorithms are well-known within the field of digital image analysis, and there exists numerous algorithms depending on the purpose. A common procedure is the Hough transformation, Hough [13], who came up with the idea of shape detection of lines based on a parameter space. The work was further developed by Duda \& Hart [14] where the shape detection was extended to include circular objects. This method is commonly referred to as the Circular Hough Transformation, and a more profound presentation can be found in Shapiro et al. [15]. The magnitude of the parameter space for the shape detection depends on the shapes considered. For instance, line detection uses a 2 parameter space (slope and intersection), circle detection 3 (centre $(x, y)$ and radius), and ellipses 5 (minor/major axis, centre $(x, y)$, and orientation). Increasing the parameter space heavily increases the computational requirements. The current method is based on the Circular Hough Transformation for detection of circular shapes.

The analysis is split up into three different steps: pre-processing, processing, and post-processing as presented in Fig. 1 and the following sub-sections. 


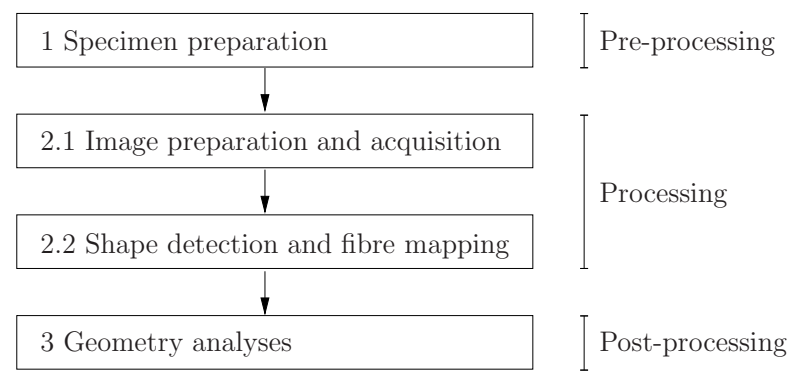

Figure 1: Flowchart for detection of composite micro-structure.

\section{$2.1 \quad$ Pre-processing}

The preparation of the test samples is crucial in order to obtain the best possible image quality. Standard microscope specimens are used where a representative material sample of the composite laminate is cut and cast in an epoxy resin to form a cylinder with a diameter of $30 \mathrm{~mm}$. Thereupon, the specimens are polished in a grinder with abrasive paper varying from \#250 to \#4000 in grain size. The polishing time is adjusted according to the grain size. It is evident that the sample surface appears as plane and smooth as possible.

\subsection{Processing}

To obtain the best image quality, it is recommended to use a Scanning Electron Microscope (SEM), an Environmental Scanning Electron Microscope (ESEM), or a Low Vacuum Scanning Electron Microscope (LVSEM) for image acquisition. If a SEM is used, the specimens need to be coated due to their non-conducting surface. It is possible to use an optical microscope, but this requires even more sensitivity in the pre-processing step due to the lower resolution, lower depth-of-field, and poorer lighting conditions in an optical microscope compared to an electron microscope. In the present work a LVSEM is used.

The magnification of the microscope should be adjusted so the misinterpretation in the radii detection is reduced, see further discussion in Section 2.4. To minimise computational requirements, the shape detection is based on 8-bit grey scale images. Upon acquisition of the micrographs, the shape detection is divided into two independent steps: a Canny edge detection of the image, Canny [16], and a circle detection based on these edges using the Circular Hough Transformation. The Canny edge detection locates edges by searching for a local maxima of the colour gradient within the considered image. An example is illustrated in Fig. 2 where the grey level intensity is illustrated along a given path in the image. It is evident that the edge of 
the fibres are detectable due to the obvious colour gradient.
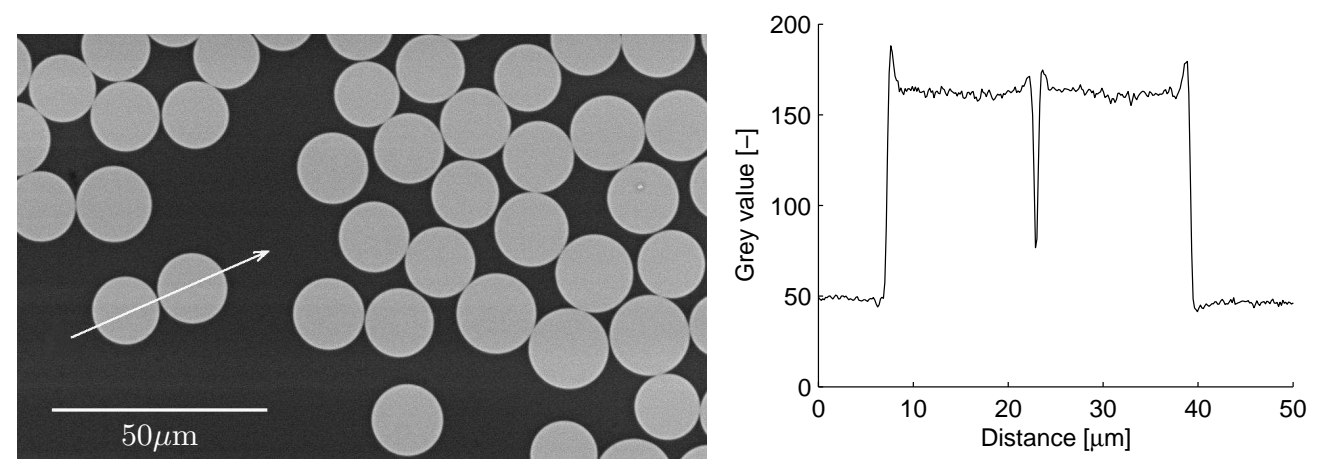

(a) SEM micrograph of glass fibres (light part) and matrix (darker part) in a UD reinforced composite. The arrow indicates the direction for the grey level intensity in Fig.

(b) Grey level intensity along the path shown in Fig. 2(a) White level is 255, black is 0 . Note that the interfacial region gives rise to a larger grey value than the fibre. $2(\mathrm{~b})$

Figure 2: Identification of fibre edges in a uni-directional (UD) fibre reinforced composite based on a SEM micrograph.

Once the edges of the fibres are detected, the Circular Hough Transformation is applied by a voting procedure to identify the best matching circles. The input parameters for the transformation can be adjusted depending on the image quality (threshold level, search range, and magnitude of search filter). The results of the detection are the in-plane fibre location ( $x, y$-coordinates) and the fibre radius, $r$.

Due to the numerical processing power there is a limit for the image size that can be analysed by the Circular Hough Transformation. Therefore, for larger image sizes (approximately larger than $1000 \times 1000$ pixels) the entire image is split up into a number of user defined segments that are analysed separately, and the results are stored for each segment. An average value for the entire image is evaluated once all segments have been analysed. The segmentation procedure can also be used to characterise the micro-structure across a fibre bundle, effects of clustering due to stitching tension, etc.

\subsection{Post-processing}

Circle sets on a plane are defined if the positions and radii of the circles are known. Characterising these circle sets requires knowledge about the number of circles, circle diameter distribution, communal distance between the circles, clustering, packing pattern, and circles in contact. When these parameters are known it is possible to make a full characterisation of the circle set. The following analyses are considered based on the fibre centre and radii obtained from the Circular Hough Transformation: 
- global fibre volume fraction

- void content

- fibre diameter distribution

- number of contact points per fibre

- nearest neighbour distance

- fibre clustering parameter

- number of neighbours and local fibre volume fraction.

Each of the above analyses are outlined in the following.

Fibre volume fraction The FVF is considered a fundamental parameter, which is used in characterising and calculating mechanical properties of fibre reinforced composites. In the present work, the FVF is determined using three distinct procedures:

1. Knowing the individual fibre radii and the total image size, it is possible to determine the FVF of the detected fibres. It is assumed that the position and diameter of the fibres do not vary in the normal direction of the fibres. Hence, the FVF is determined as:

$$
\mathrm{FVF}=\frac{\sum_{i=1}^{N} V_{i, f i b r e}}{V_{\text {total }}}=\frac{\sum_{i=1}^{N} A_{i, f i b r e}}{A_{\text {image }}}=\frac{\pi \sum_{i=1}^{N} r_{i}^{2}}{A_{\text {image }}}
$$

where $V$ is the volume, $A$ is the area, $N$ is the number of fibres, and $r_{i}$ is the individual fibre radius. This procedure for determining the FVF is referred to as FVF1.

2. A simple procedure to evaluate the FVF is by a threshold analysis. The image is transformed into a black/white image whereas the resin appears as black pixels and the fibres as white pixels. The FVF is found as the ratio between the white pixels and the total number of pixels. This procedure is referred to as FVF2.

3. It is likely that the Circular Hough Transformation does not detect every fibre in the cross-section especially near the image boundary where the fibres are cut off. This procedure simply removes the detected fibres (used in procedure one) and performs a threshold analysis (as in procedure two) on the remaining undetected fibres. This procedure is referred to as FVF3.

It is given that the sum of the FVF's from procedures one and three should match procedure two. 
Void content Voids may be present in the materials, and these voids are known to influence the mechanical properties. Since voids appear as black regions in the micrographs, these are identified if a pixel value is lower than a user defined threshold. The total void content is found as the sum of these pixels in relation to the total number of pixels.

Fibre diameter distribution It is often assumed in numerical analyses of fibre reinforced composites that the fibre diameter distribution is uniform, which is often not the case in practice. The fibre diameter is measured by the Circular Hough Transformation and assumed normal distributed meaning that it is characterised by the mean value and the standard deviation.

Number of contact points per fibre Contact between surfaces leads to stress concentrations; therefore, contact between fibres is considered as a potential zone for crack initiation, see e.g. Wongsto \& Li [10]. A contact condition between two circles (fibres) is defined if the centreto-centre distance between two adjacent circles $i$ and $j, d_{i j}$, is less than the sum of their individual radii, $r_{i}+r_{j}$. However, due to the fibre surface roughness, interface, and inaccuracy in the circle detection, the contact condition is given as $d_{i j} \leq\left(r_{i}+r_{j}\right) \alpha$ where the factor $\alpha=1.01$ follows from the work of Mishnaevsky \& Brøndsted [12. Denoting the total number of circle contact conditions as $c$, the total number of contact points, $\mathrm{CP}_{\text {total }}$, is determined as:

$$
\mathrm{CP}_{\text {total }}=\frac{c-N}{2},
$$

where the factor of two is included to avoid repeated contact points. Since the total number of contact points in Eq. (2) is dependent on the number of inclusions, the total number of contact points is normalised with the number of detected fibres, $N$, in order to get the number of contact points per fibre, CP. For two common fibre packing patterns, the number of contact points per fibre and FVF is illustrated in Fig. 3 for an infinite fibre array. 


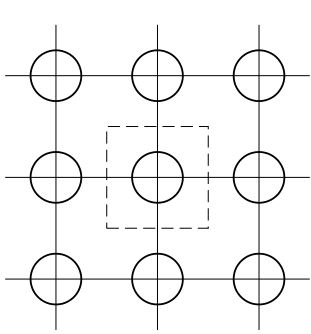

(a) Square array.
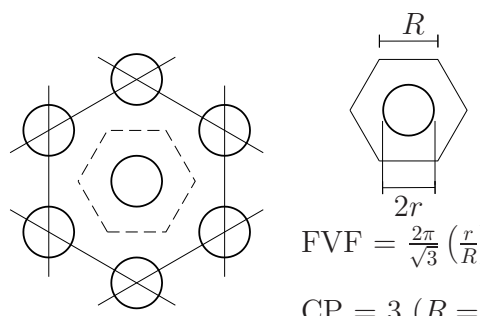

$\mathrm{FVF}=\frac{2 \pi}{\sqrt{3}}\left(\frac{r}{R}\right)^{2}$

$\mathrm{CP}=3(R=2 r)$

(b) Hexagonal array.

Figure 3: Typical fibre packing patterns in a uni-directional composite along with fibre volume fraction (FVF) and number of contact points per fibre $(\mathrm{CP})$.

Nearest neighbour distance Separation distance between the fibres may minimise the stress concentration, and this analysis determines the distance to the nearest neighbour. The Neighbouring Distance between fibres $i$ and $j$ is defined as $\mathrm{ND}_{i j}=d_{i j}-r_{i}-r_{j}$, and the shortest distance is found by the solution to the classical Travelling Salesman Problem (TSP) using a TSP-algorithm. Further information on the TSP can be found e.g. in Lawler et al. [17. The result of the analysis is the "shortest route" through all fibres, and thereby the Nearest Neighbouring Distances, NND. For quantification, the nearest neighbour distance is assumed to follow a log-normal behaviour with mean $\mu$ and standard deviation $\sigma$ meaning that the limits are expressed as $\exp (\log (\mu) \pm \log (\sigma))$.

Fibre clustering Clustering of fibres leads to regions that influence the mechanical behaviour. In order to estimate the fibre clustering, the simple relation due to Clark \& Evans [18] is used as a measure for the fibre clustering. Originally, the theory was used in terms of spatial relations in populations, but it is directly applied in the current study even though there are some limitations. The clustering parameter, $R$, is expressed as:

$$
R=\frac{\bar{r}_{A}}{\bar{r}_{E}} \quad, \quad \bar{r}_{A}=\frac{\sum \mathrm{NND}}{N} \quad \text { and } \quad \bar{r}_{E}=\frac{1}{2 \sqrt{\rho}}
$$

with $\bar{r}_{A}$ being the mean of the series of distances to the nearest neighbour, and $\bar{r}_{E}$ is the mean distance to the nearest neighbour expected in an infinitely large random distribution with density $\rho$. In the current case, the sum of the nearest neighbouring distances, $\sum N N D$, is found as the "shortest route" between fibre centre mentioned above. The fibre density is found as $\rho=N / A_{\text {image }}$. 
The convenient thing about the clustering parameter is its easily accessible interpretation since it is bound by the limits $0 \leq R \leq 2.15$ where the lower limit follows from an intermediate neighbouring distance equal to zero (fully clustered) and the upper limit is for a hexagonal array with equidistant distance to other neighbours. For $R=1$ the packing pattern is random.

Number of neighbours and local fibre volume fraction Often when modelling the composite micro-structure an idealised fibre packing pattern is assumed to be in the arrays shown in Fig. 3, These packing patterns are seldomly found in practice, and the following analysis estimates the packing pattern in terms of the number of neighbours and a local fibre volume fraction. More sophisticated methods may be used to characterise the packing pattern e.g. the second order intensity function as proposed by Pyrz [9] and Ghost et al. [19].

The number of neighbours is found from a Delaunay triangulation of the fibre centre points, and the local FVF is determined as the fibre area in relation to the area of Voronoi cell associated to each fibre. In brief, a Voronoi tessellation is a decomposition of a scatter (in this case points on the plane) into a cell structure each containing exactly one point. The property of the cell is that any point inside the cell is closer to that point than to any other site. A Delaunay triangulation, on the other hand, is sort of the dual problem to the Voronoi tessellation such that no point sets are inside the circumcircle of any triangle. The two principles are illustrated for a generated fibre arrangement in Fig. 4. For a more profound presentation see Okabe [20].

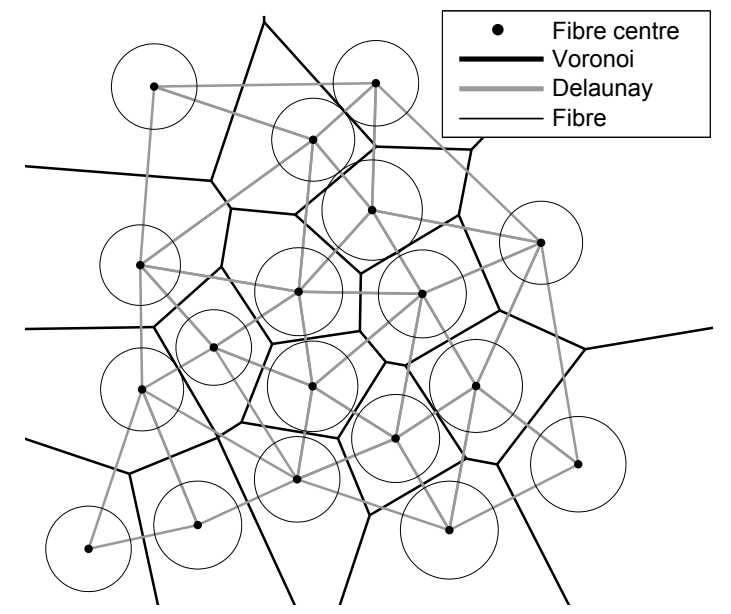

Figure 4: Sketch of the Voronoi tessellation and Delaunay triangulation for a random point set in the plane. 
In the present analysis, the Voronoi cells are used to determine a local FVF (referred to FVF4) for each fibre, since the area of the cell, $A_{\text {voronoi }}$, can be considered as an 'area parameter' associated to each fibre with area, $A_{\text {fibre }}$. Thereby, the local FVF is determined as $\frac{A_{\text {fibre }}}{A_{\text {voronoi }}}$. The area of the Voronoi cells along the image boundary cannot be evaluated explicitly, which is why the boundary fibres are disregarded. The local FVF is assumed to follow a normal distribution described by the mean value and the standard deviation. The centre points of the detected fibres are used as input for the triangulation in order to find the number of neighbours, here presented as the mean value and standard deviation, respectively. Similar analyses have been used previously e.g. in the work of Paluch [2].

\subsection{Implementation}

The programming language MATLAB is used for the implementation of the methodology. The Circular Hough Transformation and the TSP-algorithm can be found at the MathWorks File Exchange [21.

The algorithm and implementation have been tested on two selected fibres on several images with different microscope magnification in order to estimate the accuracy of the circle detection. The fibres are shown in Fig. 5(a) where Fibre A is completely isolated, and Fibre B is surrounded by several others. The result from the analysis is shown in Fig. 5(b) where the detected fibre diameter is plotted as function of microscope magnification. 


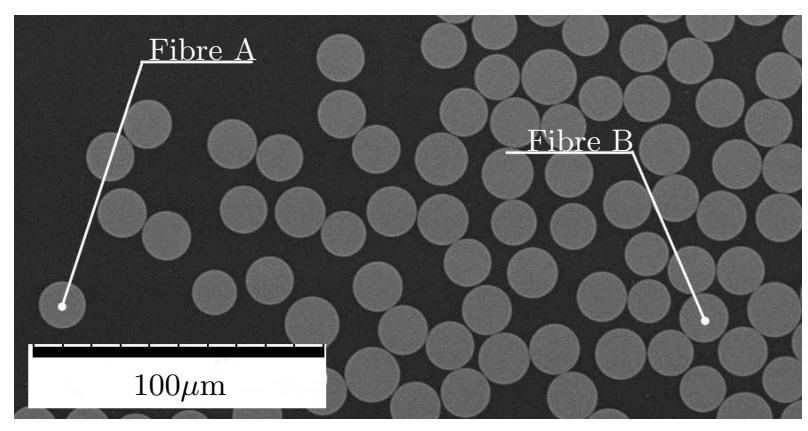

(a) Fibres considered in the accuracy analysis.

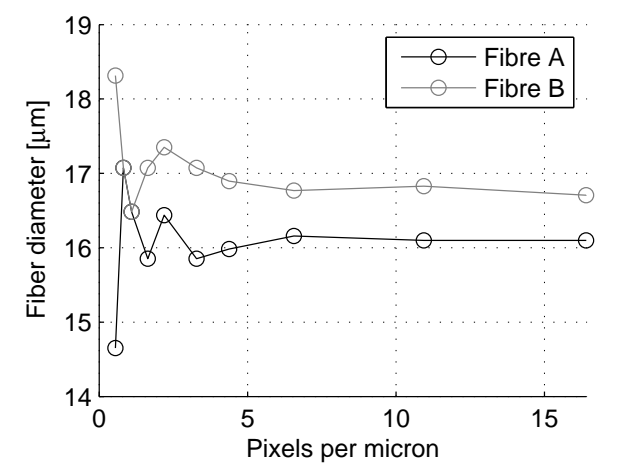

(b) Detected fibre diameter as a function of microscope magnification.

Figure 5: Accuracy analysis for detection of the fibre radius.

For low magnification images below 5 pixels per micron there is a scatter in the measured fibre diameters. However, in order to get sufficient accuracy and amount of fibres per image, a value of 1.64 pixels per micron is used in the following well aware that this gives rise to a non-negligible deviation in determination of the fibre volume fraction in procedure FVF1 mentioned above.

Depending on the image shape, image size, and number of fibres, the number of contact points is affected. Therefore, a test is carried out to investigate the image shape/size sensitivity. The test is carried out for a square fibre packing arrangement with no intermediate distance between the fibres. For a varying image shape/size, it turns out that for a 'low' number of fibres (say, less than 50 fibres in a narrow shaped image) the number of contact points is affected. 


\section{Application}

Two-layered glass/polyester composites with varying fibre content were manufactured using the Vacuum Assisted Resin Transfer Moulding (VARTM) process, and the specimens are analysed by the methodology outlined in Section 2. All images are acquired within a bundle without any resin rich zones near the edges. A typical result of the circle detection is presented in Fig. 6,

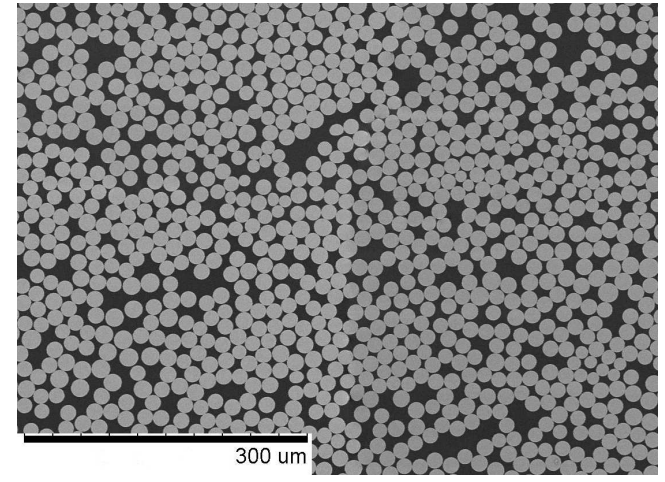

(a) Basis for image analysis: SEM micrograph of a polished cross-section.

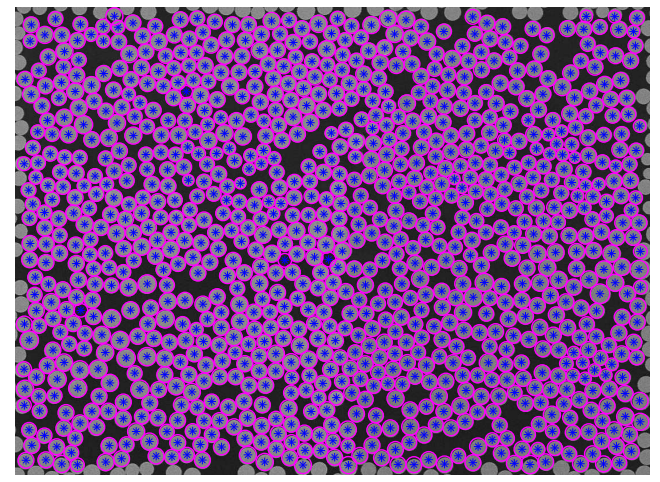

(b) Spatial mapping of the fibres with detected radii using the Circular Hough Transformation.

Figure 6: Typical images for analysing the micro-structure of a unidirectional glass fibre reinforced composite. The presented images are segments of the analysed images.

Close examination of the detected circles shown in Fig. 6(b) reveals that the algorithm detects a number of non-existing fibres; nonetheless, these fibres are removed in the post-processing step by evaluation of the grey level intensity at the centre of the (mis)detected fibre. The post-processing analyses are carried out as mentioned in the previous section and the results are presented in Table 1 and Figs. 7 and $8, \mu$ and $\sigma$ denote the mean value and standard deviation, and all data are assumed to be normal/log-normal distributed and independent. Table 1 presents the number of detected fibres and the fibre diameter distribution. 
Table 1: Result of image analysis: number of detected fibres and fibre diameter distribution. Mean $\mu$ and standard deviation $\sigma$.

\begin{tabular}{ccc}
\hline $\begin{array}{c}\text { No. } \\
\text { NoF } a \\
{[-]}\end{array}$ & $\begin{array}{c}\text { FD } \mu / \sigma \\
{[\mu \mathrm{m} / \mu \mathrm{m}]}\end{array}$ \\
\hline 1 & 4745 & $17.19 / 1.46$ \\
2 & 4605 & $17.17 / 1.45$ \\
3 & 4797 & $17.21 / 1.57$ \\
4 & 3335 & $16.96 / 1.44$ \\
5 & 7679 & $17.32 / 1.50$ \\
\hline & & \\
\hline \multirow{2}{*}{$\begin{array}{l}\text { NoF } \\
\text { FD }\end{array}$} & \multicolumn{2}{l}{ Number of Fibres } \\
\end{tabular}

Fig. 7 presents the obtained FVF's for the different analyses, and the results are normalised with the value from FVF3 (threshold analysis) in sample number one.

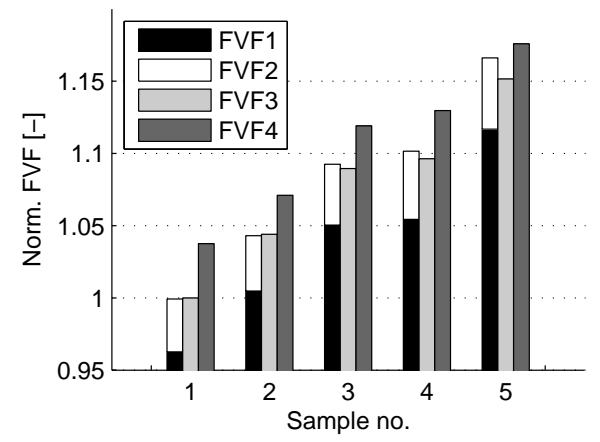

Figure 7: Results of image analyses: fibre volume fraction based on different procedures. FVF1: radii of fibres. FVF2: boundary analysis. FVF3: threshold analysis. FVF4: local (Voronoi).

Fig. 8 shows the number of contact points per fibre, CP, the nearest neighbour distance, NN, the clustering parameter, ClP, and the number of neighbours, NoN as a function of the obtained FVF. Again, the FVF is normalised with the value from FVF3 (threshold analysis) in sample number one. 
(a)

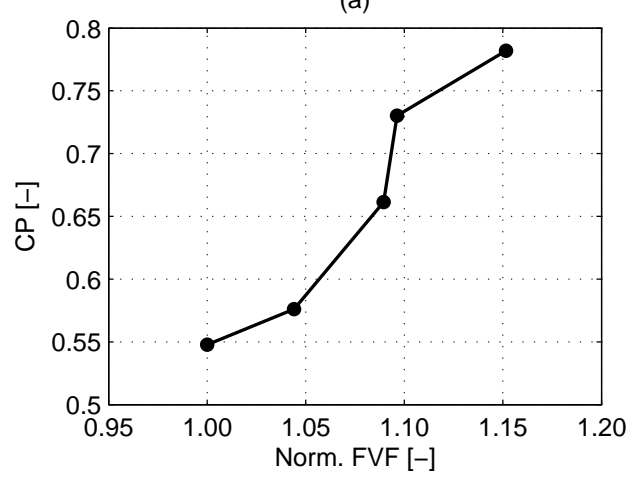

(c)

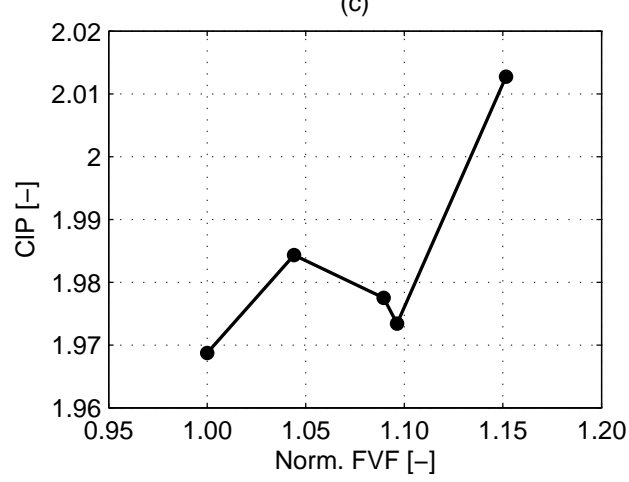

(b)

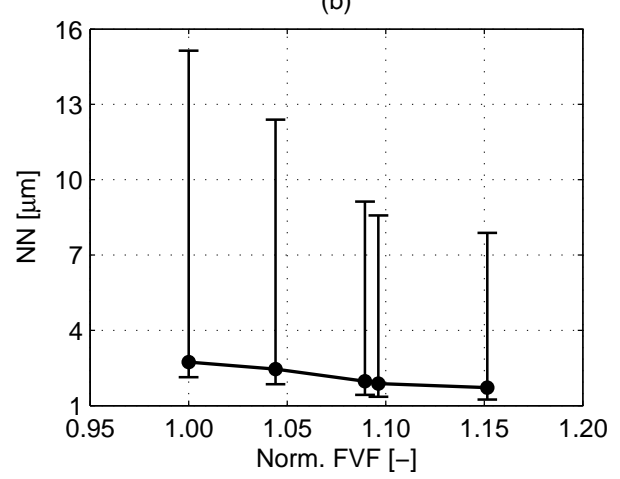

(d)

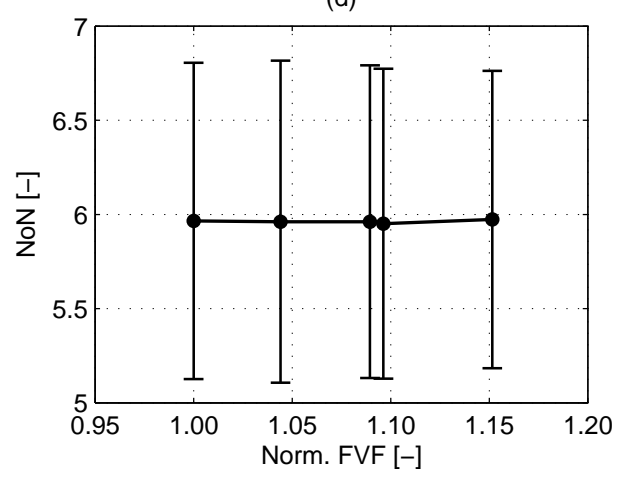

Figure 8: Results of image analyses for different FVF's. (a) Contact points per fibre, CP. (b) Nearest neighbour distance, NN. (c) Clustering parameter, ClP. (d) Number of neighbours, NoN.

The variation in the fibre diameters is small, and the measurements are almost constant in the range around $d_{f} \simeq 17 \mu \mathrm{m}$, which is also the prescribed diameter by the manufacturer.

As mentioned in the previous section, the sum of the FVF's determined from procedures FVF1 and FVF2 should be equal to the FVF determined by procedure FVF3. This is illustrated in Fig. 7 where the deviation between the methods is within $\pm 1.2 \%$, which is regarded as a sufficient accuracy in the shape detection. It is worth noticing that the local fibre volume fraction, FVF4, is consistently larger than the FVF's determined from the other analyses. No voids are found in the samples investigated.

For a heavier fibre compaction, it is found that the number of Contact Points per fibre (CP) increases as well as the Nearest Neighbour distance (NN) decreases, see Fig. 8. The packing pattern remains the same independent of the FVF, which is reflected in the Number of Neighbours (NoN) and the Clustering Parameter (ClP) approaching the upper limit of 2.15. This means that the packing pattern converges against a pseudo-hexagonal array. 


\section{Discussion}

From the number of fibres detected (see e.g. Table 1) and the image sizes used, the image size sensitivity in relation to the number of contact points per fibre is limited for the samples considered. The number of contact points per fibre increases for increasing fibre volume fraction, which in accordance to what is reported by Mishnaevsky \& Brøndsted [12] in a numerical study. Even though the actual fibre packing in Fig. 6(a) does not appear to be systematic, the fibres tend to arrange in what is referred to as a pseudohexagonal packing pattern. Using the Delaunay triangulation, Paluch [2] made a similar conclusion in relation to the packing pattern.

Pyrz 9] carried out a quantitative study on the micro-structure of composites based on statistical analyses. In specific, a probability investigation was made between the nearest neighbouring distance for different FVF's. The trend is obviously that the larger FVF, the smaller a mean nearest neighbouring distance and standard deviation is observed. The same findings are concluded in the present study.

The clustering parameter described in the work of Clark \& Evans 18 is determined based on the fibre centre without information regarding the fibre radius. Therefore, the basis of using this analysis is misleading since the underlying statistical theory is based on point sets rather than circles. It has not been possible at this stage of the study to find a suitable description for clustering of circles where the individual radii are included.

It is apparent that it would be more accurate to determine the Voronoi diagrams in terms of circle sets on a plane rather than points due to the circular cross section of the fibres, see e.g. presentation by Kim et al. [22]. However, based on the work of Paluch [2] and to ease the implementation, the presented method is considered to be sufficient.

The local FVF (FVF4) predicts a larger value compared to the global FVF, which is in contradiction to what could be expected from the idealised fibre arrangements in Fig. 3 that produces similar results for a local and global FVF. Still, the magnitudes of the deviation is in similar range to what is found from Ghosh et al. [19] based on simulations of uniform fibre distributions with FVF in the range up to $32.4 \%$.

The optimum solution to the 'travelling salesman problem' has been investigated by several researchers, and there exist numerous of different solution techniques for the problem. However, the problem of finding the optimum solution is far from trivial. In the case of $N$ fibres, the number of different routes is given as $\frac{(N-1) !}{2}$, which indicates the increasing problem complexity for large $N$. Therefore, approximated methods are often used where the route might not be the optimum one but somewhat close to. Such an algorithm is used in the present, and as a result the shortest route might not be the optimum. 


\section{Conclusion}

A new methodology is presented dealing with shape detection and microstructural analysis of composite materials containing circular fibres. Based on digital micrographs and numerical image processing, the fibre architecture/microstructure is evaluated for a number of different parameters. For demonstration, the methodology is shown useful to identify and to compare microstructural parameters for different glass fibre reinforced composites with varying fibre content. The following micro-structural parameters are affected by changes in the fibre volume fraction: the number of contact points per fibre, nearest neighbour distance, and the local fibre volume fraction (the fibre area in relation to the area of the associated Voronoi cell). For increasing fibre volume fraction, the number of contact point per fibre increases whereas the nearest neighbour distance decreases. The local fibre volume fraction is somewhat larger than the global fibre volume fraction. Voids are not found in the samples. Independent of the fibre volume fraction, the fibres are arranged in a pseudo-hexagonal array with approximately six neighbours per fibre.

\section{References}

[1] R. M. Jones. Mechanics of composite materials. Hemisphere Pub, 1999.

[2] B. Paluch. Analysis of geometric imperfections affecting the fibers in unidirectional composites. Journal of composite materials, 30(4):454, 1996.

[3] A. R. Clarke, G. Archenhold, and N. C. Davidson. A novel technique for determining the 3D spatial distribution of glass fibres in polymer composites. Composites science and technology, 55(1):75-91, 1995.

[4] S. W. Yurgartis. Techniques for the quantification of composite mesostructure. Composites science and technology, 53(2):145-154, 1995.

[5] C. J. Creighton, M. P. F. Sutcliffe, and T. W. Clyne. A multiple field image analysis procedure for characterisation of fibre alignment in composites. Composites Part A: Applied Science and Manufacturing, 32(2):221-229, 2001.

[6] K. K. Kratmann, M. P. F. Sutcliffe, L. T. Lilleheden, R. Pyrz, and O. T. Thomsen. A novel image analysis procedure for measuring fibre misalignment in unidirectional fibre composites. Composites Science and Technology, 69(2):228-238, 2009. 
[7] H. E. Exner. Stereology and 3D microscopy: Useful alternatives or competitors in the quantitative analysis of microstructures? Image Analysis \& Stereology, 23(2):73-82, 2004.

[8] F. J. Guild and J. Summerscales. Microstructural image analysis applied to fibre composite materials: a review. Composites, 24(5):383-393, 1993.

[9] R. Pyrz. Quantitative description of the microstructure of composites. Part I: Morphology of unidirectional composite systems. Composites science and technology, 50(2):197-208, 1994.

[10] A. Wongsto and S. Li. Micromechanical FE analysis of UD fibrereinforced composites with fibres distributed at random over the transverse cross-section. Composites Part A: Applied Science and Manufacturing, 36(9):1246-1266, 2005.

[11] A.R. Melro, P.P. Camanho, and S.T. Pinho. Generation of random distribution of fibres in long-fibre reinforced composites. Composites Science and Technology, 68(9):2092-2102, July 2008.

[12] L. Mishnaevsky Jr. and P. Brøndsted. Statistical modelling of compression and fatigue damage of unidirectional fiber reinforced composites. Composites Science and Technology, 69(3-4):477-484, 2009.

[13] P. V. C. Hough. Method and means for recognizing complex patterns. Technical report, 1962.

[14] R. O. Duda and P. E. Hart. Use of the Hough transformation to detect lines and curves in pictures. Communications of the ACM, 15(1):11-15, 1972 .

[15] L. G. Shapiro and G. C. Stockman. Computer Vision. 2000.

[16] J. Canny. A computational approach to edge detection. Pattern Analysis and Machine Intelligence, IEEE Transactions on, (6):679-698, 1986.

[17] E. L. Lawler, A. H. Rinnooy-Kan, J. K. Lenstra, and D. B. Shmoys. The traveling salesman problem: a guided tour of combinatorial optimization, volume 3. Wiley New York, 1985.

[18] P. J. Clark and F. C. Evans. Distance to nearest neighbor as a measure of spatial relationships in populations. Ecology, 35(4):445-453, 1954.

[19] S. Ghosh, Z. Nowak, and K. Lee. Quantitative characterization and modeling of composite microstructures by Voronoi cells. Acta Materialia, 45(6):2215-2234, June 1997. 
[20] A. Okabe. Spatial tessellations: concepts and applications of Voronoi diagrams. John Wiley \& Sons Inc, 2000.

[21] MathWorks Inc. MATLAB Central - Files. March 2011.

[22] D. S. Kim, D. Kim, and K. Sugihara. Voronoi diagram of a circle set from Voronoi diagram of a point set: II. Geometry. Computer Aided Geometric Design, 18(6):563-585, 2001. 